\title{
Utilización de efluentes urbanos tratados para la producción de brócoli mediante riego por goteo subterráneo
}

\author{
Salusso, F., Crespi, R., Ramos, D., Pautasso, G. y Bouzo, C.
}

\begin{abstract}
RESUMEN
La utilización de efluentes constituye una alternativa viable en la producción agrícola, transformando un desperdicio en un recurso útil, aportando agua y nutrientes a los cultivos. El objetivo del estudio fue evaluar la utilización de efluentes urbanos tratados en la producción de brócoli (Brassica oleracea var. itálica). Los tratamientos resultaron de la combinación de tres cultivares: 'Matsuri' (C1), 'Green Pia' (C2) y 'Almanor' (C3), y dos fuentes de riego: efluentes urbanos tratados (EUT) y agua de acuífero (AC), suministrado mediante riego por goteo subterráneo durante dos temporadas (E1 y E2). El diseño experimental fue en parcelas divididas. A la cosecha se evaluó partición de asimilados en hojas, tallo y pella, diámetro de pella, rendimiento fresco y calidad sanitaria del producto mediante análisis microbiológicos y parasitológicos. Los resultados determinaron diferencias significativas ( $p \leq 0,05$ Fisher) en rendimiento para los cultivares C2 y C3 (E1) regados con EUT. El diámetro de pellas presentó diferencias para C2, C3 (E1) y C3 (E2). En E1 las condiciones ambientales fueron más favorables, influyendo en el rendimiento de los cultivares. La calidad sanitaria de las pellas determinó que fueron aptas para su consumo en fresco, lo que demuestra que esta tecnología podría ser utilizada en la producción de brócoli.
\end{abstract}

Palabras clave: efluentes urbanos tratados, brócoli, riego por goteo subterráneo, calidad sanitaria.

Salusso, F., Crespi, R., Ramos, D., Pautasso, G. and Bouzo, C., 2019. Use of treated urban effluents for subsurface drip irrigation in the production of broccoli. Agriscientia 36: 63-71

\section{SUMMARY}

The use of effluents constitutes a viable alternative in agricultural production, transforming a waste into a useful resource, providing water and nutrients to crops. The objective of the study was to evaluate the use of treated urban 
effluents in the production of broccoli (Brassica oleracea var. italica). The treatments resulted from the combination of three cultivars: 'Matsuri' (C1), 'Green Pia' (C2) and 'Almanor' (C3), and two irrigation sources: treated urban effluents (EUT) and aquifer water (AC), supplied by subsurface drip irrigation during two seasons (E1 and E2). The experimental design was in divided plots. At harvest, partition of assimilates in leaves, stems and head was evaluated as well as head diameter, fresh yield and sanitary quality of the product through microbiological and parasitological analyses. The results showed significant differences ( $p \leq 0.05$ Fisher) in yield for cultivars C2 and C3 (E1) irrigated with EUT. The head diameter presented differences for C2, C3 (E1) and C3 (E2). In $\mathrm{E} 1$ the environmental conditions were more favorable, influencing the yield of the cultivars. The sanitary quality tests of the heads determined that they were adequate for fresh consumption, demonstrating that this technology could be applied for broccoli production.

Keywords: treated urban effluents, broccoli, subsurface drip irrigation, sanitary quality.

Salusso, F., Ramos, D. y Pautasso, G.: Depto. de Producción Vegetal, Facultad de Agronomía y Veterinaria, Universidad Nacional de Río Cuarto. Ruta No $36 \mathrm{Km}$ 601, 5800, Río Cuarto, Córdoba. Tel. 0358-4676159. Crespi, R.: Depto de Ecología Agraria, Facultad de Agronomía y Veterinaria, Universidad Nacional de Río Cuarto. Bouzo, C.: Depto de Producción Vegetal, Facultad de Ciencias Agrarias, Universidad Nacional del Litoral, Luis Kreder 2805, Esperanza, Santa Fe. Correspondencia a: fsalusso@ayv.unrc.edu.ar

\section{INTRODUCCIÓN}

La utilización de aguas residuales tratadas es una práctica común en muchos países de regiones áridas y semiáridas donde el agua dulce es fundamental para el desarrollo urbano (Beneduce et al., 2017). El uso de aguas residuales en agricultura constituye una herramienta valiosa para reducir la contaminación ambiental y enfrentar el desafío de incrementar la producción agrícola con un recurso hídrico escaso (Sáenz Forero, 2006). En muchos lugares, los efluentes urbanos son vertidos directamente al cauce de los ríos, contaminando estos cursos de agua y provocando su eutroficación, y aumentando el riesgo de trasmisión de enfermedades a la población humana (Crespi, Camacho y Polo, 2009; Disciglio et al., 2015). El uso de efluentes en sistemas agrícolas no solo garantiza una fuente constante y segura de agua, sino también un aporte de nutrientes para los cultivos (González y Rubalcaba, 2011; Moazeni et al., 2017), posibilitando un importante ahorro en la utilización de fertilizantes de síntesis química (Tran, Schwabe y Jassby, 2016; Vergine et al., 2017). Las aguas residuales tratadas pueden contener materia orgánica fácilmente biodegradable y nutrientes disponibles como nitrógeno, fósforo y potasio, que absorbidos por las plantas aumentan la producción y rendimiento de los cultivos (Disciglio et al., 2015). Una alternativa viable puede constituir su utilización en sistemas de producción hortícola, ya que la mayoría de estos sistemas se encuentran en cercanías de los centros poblados donde se generan aguas residuales. Algunas experiencias demuestran que su aplicación en cultivos de tomate (Navarro López, 2010; Orlofsky et al., 2016), brócoli (Beneduce et al., 2017), zucchini (Nieto et al., 2001) y ajo (Ramos et al., 2016), permitieron obtener resultados beneficiosos. No obstante, aunque constituyen un recurso valioso, los efluentes contienen microorganismos patógenos como Escherichia coli (Ackers et al., 1998; Hilborn et al., 1999), Salmonella sp. (Isaacs et al., 2005) y diferentes tipos de parásitos (Reinoso, Torres y Becares, 2008). Por este motivo se debe procurar un adecuado manejo y tratamiento del efluente crudo para minimizar el riesgo que implican estos microorganismos para la salud humana (Fasciolo, Meca, Calderon y Rebollo, 2005). La aplicación de efluentes urbanos mediante sistemas de riego subterráneos, presenta una gran ventaja al posicionar el agua y los nutrientes en inmediaciones de las raíces de los cultivos, haciendo un uso más eficiente y menos riesgoso del recurso (Lanchimba Morales, 2016). La búsqueda de fuentes alternativas de agua es un factor determinante para 
incrementar la productividad en sistemas agrícolas y contribuir a su desarrollo sustentable. En este contexto, el objetivo del estudio fue demostrar la viabilidad de utilizar efluentes urbanos tratados en cultivo de brócoli aplicados mediante un sistema de riego por goteo subterráneo.

\section{MATERIALES Y MÉTODOS}

Los experimentos se realizaron en el campo experimental de la Universidad Nacional de Río Cuarto (UNRC), Córdoba (Argentina), en una Planta Piloto de Tratamiento y Reutilización de Efluentes

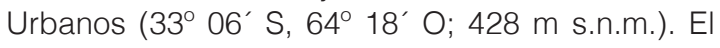
estudio se desarrolló mediante dos experimentos (E1 y E2) realizados en años sucesivos, evaluando tres cultivares de brócoli (Brassica oleracea var. italica): 'Matsuri' F1 (C1), 'Green Pia' F1 (C2) y 'Almanor' F1 (C3), regados con dos calidades de agua: efluentes urbanos tratados (EUT) y agua de acuífero (AC).

En E1 la siembra de los cultivares se realizó el día 28/03/2016 y en E2, el 18/03/2017, en bandejas de germinación de 200 celdas $\left(13 \mathrm{~cm}^{3}\right.$ celda $^{-1}$ ), utilizando como sustrato turba Sphagnum. Durante el crecimiento inicial de las plántulas las bandejas se situaron en invernadero. El transplante a campo, se efectuó a los 50 (E1) y 52 (E2) días después de la siembra, a una densidad de 4 plantas $\mathrm{m}^{-2}$, y en un marco de plantación de 0,50 m entre hileras y entre plantas sobre la hilera.

Los efluentes crudos que se utilizaron para riego provinieron de un complejo de residencias estudiantiles ubicado en el predio de la Universidad Nacional de Río Cuarto, y para su tratamiento se recolectaron y condujeron mediante una red de tuberías hacia una cámara receptora de 3000 L para un filtrado inicial. Desde allí los efluentes fueron derivados hacia un tanque sedimentador con una capacidad de $10000 \mathrm{~L}$, cuya función fue concentrar el efluente y permitir la deposición de los sólidos contenidos en el mismo en forma de lodos para su separación.

En una segunda etapa los efluentes se extrajeron desde la parte superior del tanque sedimentador y se derivaron a una laguna facultativa (Figura 1a) con presencia de macrófitas acuáticas (Lemnas $\mathrm{sp}$.) para su depuración biológica, durante un período denominado tiempo de residencia hidráulico (TRH) de 13 días. Una vez trascurrido ese tiempo se derivaron a una segunda laguna facultativa de iguales características a la anterior, donde permanecieron un TRH de 13 días más; durante estas etapas se produce una disminución de la presencia de bacterias coliformes fecales y totales. Posteriormente, los efluentes fueron conducidos a una laguna de maduración de poca profundidad, cuya función fue reducir aún más la presencia de microorganismos a través de la exposición a la radiación solar directa (Figura 1b). Además, se realizó un seguimiento mediante análisis microbiológicos durante el proceso de depuración del EUT y del suelo en la parcela de ensayo a $5 \mathrm{~cm}$ de profundidad.

Por su parte, el agua de acuífero utilizada para riego se extrae del acuífero libre de la faja fluvial del río Cuarto, que posee aguas dulces de gran aptitud para todo tipo de usos, explotándose en este ámbito el recurso hídrico con el que se abastece a la ciudad de Río Cuarto (Blarasin, Cabrera y Matteoda, 2014).

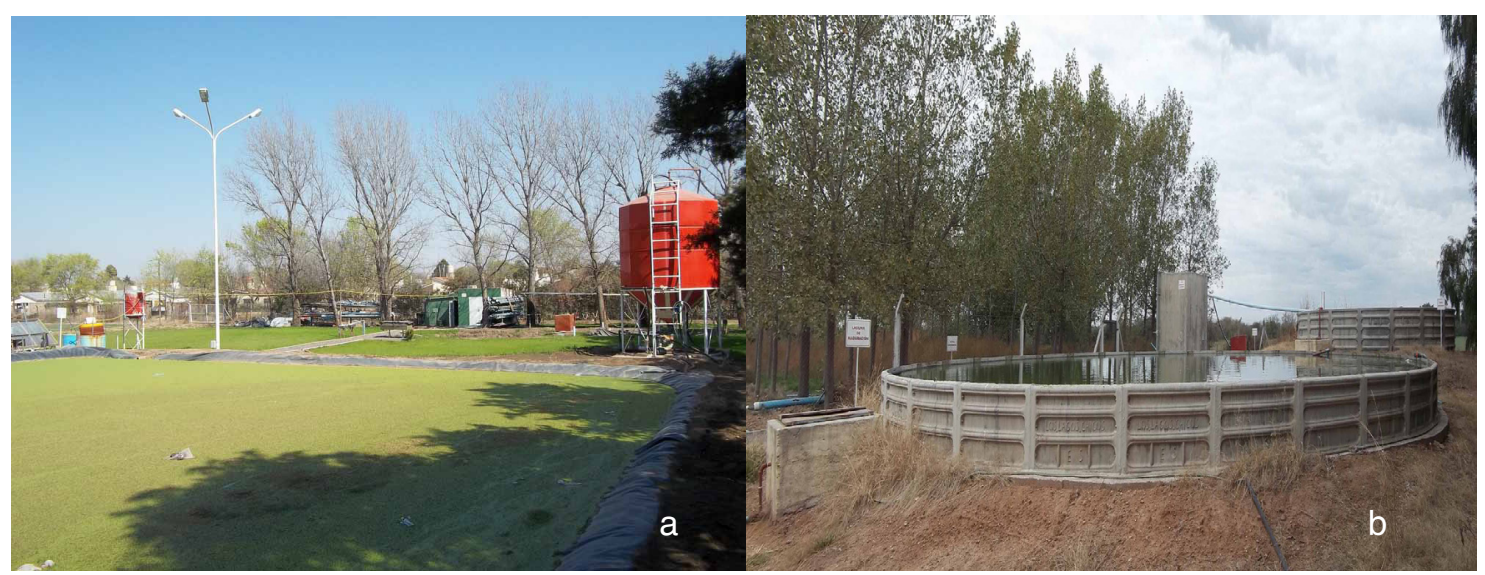

Figura 1. a) Lagunas facultativas con presencia de macrófitas acuáticas en superficie. b) Laguna de maduración. Planta Piloto de Tratamiento y Reutilización de Efluentes Urbanos, UNRC. 
El diseño de los experimentos (E1 y E2) se realizó en parcelas divididas con seis tratamientos y cinco repeticiones, constituidos por la combinación de los tres cultivares y las dos calidades de aguas de riego, siendo el factor de las parcelas principales la calidad de agua de riego, mientras que las subparcelas se correspondieron con los cultivares de brócoli. La superficie total de cada parcela principal fue de $81,0 \mathrm{~m}^{2}$ y las subparcelas de 5,4 $\mathrm{m}^{2}$. Los experimentos se realizaron sobre un suelo Haplustol típico cuyas características químicas hasta una profundidad de 0,20 $\mathrm{m}$ se presentan en la Tabla 1.

El riego de las parcelas con EUT y AC se realizó mediante un sistema de goteo subterráneo, compuesto por una red principal de tuberías de $50 \mathrm{~mm}$ de diámetro ubicadas a una profundidad de $0,40 \mathrm{~m}$, en la cual se insertaron conectores y líneas portagoteros de $16 \mathrm{~mm}$ de diámetro con emisores de una capacidad de descarga de $1 \mathrm{Lh}^{-1}$. El distanciamiento entre las líneas portagoteros fue de 0,50 $\mathrm{m}$ y se ubicaron a una profundidad de 0,20 $\mathrm{m}$. En la Tabla 2 se muestran los datos analíticos de la calidad del EUT y AC determinados en el laboratorio de Tecnología Química de la Facultad de Ingeniería de la UNRC.

Para determinar el momento de riego se midió la humedad del suelo en forma gravimétrica (Gil y Martelotto, 1993), y la programación del riego se definió evitando que el contenido de humedad sea inferior a un umbral de $60 \%$ del agua útil. A través de una estación meteorológica automática Licor modelo Li 1200 S situada en el sitio experimental se registró temperatura del aire $\left({ }^{\circ} \mathrm{C}\right)$ y precipitaciones.

La cosecha de los cultivares se realizó cuando las pellas alcanzaron la madurez óptima de consumo (Cantwell y Suslow, 1999), procediéndose a determinar la partición de asimilados en hojas, tallo y pella ( $\left.\mathrm{g} \mathrm{MS} \mathrm{planta}^{-1}\right)$, diámetro de pella $(\mathrm{mm})$ y rendimiento total $\left(\mathrm{Mg} \mathrm{ha}^{-1}\right)$. La determinación de indicadores de inocuidad sobre muestras de pellas frescas se realizó mediante análisis microbiológicos para detectar la presencia de Escherichia coli, Salmonella sp., coliformes fecales y totales (ISO, 2001; ISO, 2002; Jay, 2002). Se realizaron análisis parasitológicos para cuantificar la presencia de larvas y estructuras parasitarias, céstodos, nemátodos y ooquistes (Teuscher, 1965).

\section{RESULTADOS Y DISCUSIÓN}

En los experimentos E1 y E2 el total de lámina de riego utilizada fue de $340 \mathrm{~mm}$ y $292 \mathrm{~mm}$, respectivamente, siendo la misma para EUT y AC en cada ciclo de producción. Las precipitaciones aportaron $108 \mathrm{~mm}$ (E1) y $114 \mathrm{~mm}$ (E2), determinando un total de agua entregada a los cultivares de $448 \mathrm{~mm}$ (E1) y $406 \mathrm{~mm}$ (E2), estos valores se encuentran dentro del rango recomendado para esta especie hortícola (De Chaves, 2016). De acuerdo a la lámina aplicada y la composición físico-química de los EUT, los cultivares recibieron en E1 un aporte de macronutrientes de 246,1; 10,9

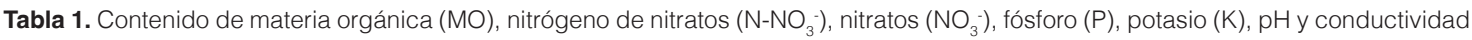
eléctrica (CE) de 0 a $20 \mathrm{~cm}$ de profundidad.

\begin{tabular}{lccccccc}
\hline Experimento & $\mathbf{M O}(\%)$ & $\mathbf{N}^{-\mathbf{N O}_{3}} \mathbf{~}^{(\mathbf{p p m})}$ & $\mathbf{N O}_{3}^{-}(\mathbf{p p m})$ & $\mathbf{P}(\mathbf{p p m})$ & $\mathbf{K}\left(\mathbf{c m o l} \mathbf{~ k g}^{-1}\right)$ & $\mathbf{p H}$ & $\mathbf{C E}\left(\mathbf{d S m}^{-1}\right)$ \\
\hline E1 & 2,23 & 24,4 & 108,3 & 37,4 & 2,01 & 7,09 & 0,25 \\
E2 & 2,01 & 15,5 & 68,8 & 44,4 & 1,77 & 6,79 & 0,17 \\
\hline
\end{tabular}

Tabla 2. Determinaciones analíticas del efluente urbano tratado (EUT) y agua de acuífero (AC).

\begin{tabular}{lccc}
\hline Determinación & Unidad & EUT & AC \\
\hline Nitrógeno total & $\mathrm{mg} \mathrm{L}^{-1}$ & 72,4 & $\mathrm{nd} \dagger$ \\
Fósforo total & $\mathrm{mg} \mathrm{L}^{-1}$ & 3,2 & $\mathrm{nd} \dagger$ \\
Potasio & $\mathrm{mg} \mathrm{L}^{-1}$ & 12,0 & 4,3 \\
Calcio & $\mathrm{mg} \mathrm{L}^{-1}$ & 50,0 & 28,4 \\
Magnesio & $\mathrm{mg} \mathrm{L}^{-1}$ & 11,6 & 9,1 \\
Sulfatos & $\mathrm{mg} \mathrm{L}^{-1}$ & 14,5 & 20,7 \\
Cloruros & $\mathrm{mg} \mathrm{L}^{-1}$ & 138,0 & 14,1 \\
C.E. & $\mathrm{dS} \mathrm{m}^{-1}$ & 1,1 & 0,4 \\
pH & --- & 7,8 & 7,4 \\
RAS & --- & 7,3 & 1,2 \\
Demanda Biológica de $\mathrm{O}_{2}$ & $\mathrm{mg} \mathrm{L}^{-1}$ & 112,6 & $\mathrm{nd} \dagger$ \\
\hline
\end{tabular}

tnd: no detectado 
y $40,8 \mathrm{~kg} \mathrm{ha}^{-1}$ de N, P y K, respectivamente. En E2 los cultivares fueron más precoces, lo que redujo las necesidades de riego, resultando los aportes de nutrientes inferiores al primer experimento, siendo estos valores de 211,$4 ; 9,3$ y $35,0 \mathrm{~kg} \mathrm{ha}^{-1}$ de $\mathrm{N}, \mathrm{P}$ y K. Algunos estudios sobre fertilización en brócoli recomiendan dosis de 145; 24 y $197 \mathrm{~kg} \mathrm{ha}^{-1}$ de $\mathrm{N}$, $\mathrm{P}$ y K para lograr un rendimiento fresco de pellas de 16,3 $\mathrm{Mg} \mathrm{ha}^{-1}$ (Lardizábal y Theodoracopoulos, 2008); otros plantean requerimientos de $90 \mathrm{~kg}$ ha $^{-1}$ de N, 15 de Py 70 de K para obtener 20 Mg ha $^{-1}$ de pellas fresca (Jaramillo y Díaz, 2006). Las demandas nutricionales pueden ser muy variables según el cultivar y condiciones ambientales de cada sitio de producción (Jaramillo y Díaz, 2006), sin embargo el aporte de nutrientes mediante el uso de EUT contribuyen a disminuir la aplicación de fertilizantes de síntesis química (Crespi et al., 2012), por lo tanto su utilización puede plantearse como una estrategia complementaria para resolver problemas de fertilización, aportar agua a los cultivos y reducir potenciales riesgos de contaminación ambiental.

El análisis de calidad del EUT determinó que sería apto para su uso en riego con restricciones ligeras o moderadas debido a la presencia de sodio, su contenido de sales y relación con el RAS (García Alvaro, 2012), cuando el agua de riego presenta una elevada concentración de sodio puede ocasionar a mediano o largo plazo, deterioros en la estructura del suelo y reducción de la permeabilidad. En este estudio, tanto en el primer como en el segundo experimento, no se observaron dificultades en la infiltración y movimiento de agua en el suelo; no obstante, es importante destacar la necesidad de realizar mediciones contínuas de las propiedades físicoquímicas del suelo ante un uso prolongado de este tipo de aguas. Algunos autores aconsejan alternar el uso de efluentes tratados con otras fuentes de agua para evitar problemas a largo plazo (Vergine et al., 2017).

Una evaluación directa del efecto de los tratamientos con EUT y AC lo proporcionó el crecimiento de las plantas. Los resultados determinaron que la MS total acumulada en cada cultivar aumentó cuando se regaron con EUT. No obstante, estas diferencias fueron solo estadísticamente significativas para los cultivares C2 y C3, siendo este último aquel que presentó la mayor respuesta con incrementos de $11,5 \%$ (E1) y $13,6 \%$ (E2). En todos los cultivares el principal componente en la partición de MS fueron las hojas, seguido de la pella y el tallo. En el cultivar C1 no hubo diferencias estadísticas en la partición de asimilados entre EUT y AC, las plantas presentaron un desarrollo foliar y tamaño de pella similares entre ambas calidades de agua.

La variable diámetro de pella respondió positivamente al riego con EUT, con diferencias más acentuadas en $\mathrm{E} 1$, donde se observaron incrementos de 4,0 (C1), 19,0 (C2) y 6,0 mm (C3), mientras que en E2 aumentó en 0,4 (C1), 4,6 (C2) y 13,3 mm (C3), aunque sólo diferenciándose significativamente en el cultivar C3.

El aporte de nutrientes a través de los EUT logró aumentar el rendimiento de pellas frescas en el orden de 33 y $20 \%$ (E1) para los cultivares C2 y C3, siendo en E2 los valores de 9 y $11 \%$, respectivamente. Los resultados se presentan en la Tabla 3.

Tabla 3. Efecto del riego con efluentes urbanos tratados (EUT) y agua de acuífero (AC) sobre tres cultivares de brócoli medido a través de la materia seca (MS) acumulada en hojas, tallo y pella (g MS planta-1), diámetro de pella (mm) y rendimiento (Mg ha $\left.{ }^{-1}\right)$ al momento de la cosecha.

\begin{tabular}{|c|c|c|c|c|c|}
\hline \multirow{2}{*}{\multicolumn{2}{|c|}{ Experimento Cultivar }} & \multirow{2}{*}{$\begin{array}{l}\text { Calidad } \\
\text { de agua }\end{array}$} & \multirow{2}{*}{$\begin{array}{c}\text { Hojas Tallo Pella Total } \\
\left(\mathrm{g} \mathrm{MS} \mathrm{planta}^{-1}\right)\end{array}$} & \multicolumn{2}{|c|}{ Diámetro de pella Rendimiento comercial } \\
\hline & & & & $(\mathrm{mm})$ & $\left(\mathrm{Mg} \mathrm{ha}^{-1}\right)$ \\
\hline \multirow{5}{*}{ E1 } & C1 & EUT & 64,1 A 20,2 A 31,5 A 115,8 A & $145 \mathrm{~A}$ & $12,43 \mathrm{~A}$ \\
\hline & & $A C$ & 65,1 A 18,1 A 29,3 A 112,5 A & $141 \mathrm{~A}$ & $11,36 \mathrm{~A}$ \\
\hline & $\mathrm{C} 2$ & EUT & 80,0 A 21,9 A 42,4 A 144,5 A & $186 \mathrm{~A}$ & $16,38 \mathrm{~A}$ \\
\hline & & $A C$ & 78,7 A 22,1 A 32,7 B 133,5 B & $167 \mathrm{~B}$ & $12,24 \mathrm{~B}$ \\
\hline & C3 & EUT & 98,6 A 30,5 A 38,2 A 167,3 A & $167 \mathrm{~A}$ & $14,73 \mathrm{~A}$ \\
\hline \multirow{7}{*}{ E2 } & & $A C$ & 89,9 A 27,2 B 32,9 B 150,0 B & $161 \mathrm{~B}$ & $12,22 \mathrm{~B}$ \\
\hline & C1 & EUT & 31,3 A 12,3 A 21,7 A 65,4 A & $125 \mathrm{~A}$ & $8,90 \mathrm{~A}$ \\
\hline & & $A C$ & 30,2 A 11,6 A 21,3 A 63,2 A & $125 \mathrm{~A}$ & $8,43 \mathrm{~A}$ \\
\hline & C2 & EUT & 34,0 A 15,6 A 17,1 A 66,8 A & $121 \mathrm{~A}$ & $7,30 \mathrm{~A}$ \\
\hline & & $A C$ & 30,4 B 12,6 B 16,0 A 59,0 B & $117 \mathrm{~A}$ & $6,70 \mathrm{~A}$ \\
\hline & C3 & EUT & 48,4 A 20,3 A 36,9 A 105,6 A & $155 \mathrm{~A}$ & $11,80 \mathrm{~A}$ \\
\hline & & $A C$ & 43,3 B 17,5 B 32,0 B 92,9 B & $142 \mathrm{~B}$ & $10,56 \mathrm{~A}$ \\
\hline
\end{tabular}

Diferentes letras en columnas de un mismo cultivar indican diferencias significativas según test de LSD Fisher ( $p \leq 0,05$ ). 
En experiencias similares otros autores no encontraron influencias significativas en la productividad de brócoli regado con efluentes tratados, obteniendo rendimientos de 7,6 y $7,5 \mathrm{Mg}$ ha-1 empleando riego con agua de perforación y efluentes tratados, respectivamente (Vergine et al., 2017). Sin embargo, se destaca la importancia de su utilización como práctica sustentable en los sistemas agrícolas.

Por otra parte, se observó para los mismos cultivares diferencias importantes en el rendimiento entre los dos experimentos. Principalmente esto se debió a que las condiciones ambientales en E2 determinaron una mayor tasa de progreso en la diferenciación de las pellas, con un menor tamaño de las plantas, produciéndose un acortamiento en el tiempo de madurez de las pellas de 15 días en comparación a E1. En E1 las temperaturas presentaron un comportamiento más estable, en cambio, en E2 existió una gran variabilidad a lo largo del ciclo de los mismos. Si el intervalo térmico es muy amplio puede afectar el normal crecimiento del brócoli (Di Benedetto, 2005); además debido a las bajas temperaturas en E2 se observó menor desarrollo foliar y mayor precocidad, llevando a un menor rendimiento en peso fresco de pellas. En la Figura 2 se muestra el comportamiento de las temperaturas máximas (línea contínua) y mínimas (línea punteada) para cada experimento.

Los resultados microbiológicos del EUT durante el proceso de depuración mostraron una notable disminución de la presencia de microorganismos patógenos, llegando a alcanzar niveles inferiores a los recomendados $\left(<1000 \mathrm{NMP} 100 \mathrm{~mL}^{-1}\right)$ por las directrices internacionales (World Health Organization, 2006; Unión Europea, 2017) para el riego de este tipo de cultivo. No obstante, las muestras de suelo indicaron la presencia de coliformes fecales, por lo cual sería necesario adoptar medidas precautorias que eviten poner en riesgo la salud de los trabajadores. Tabla 4.

La evaluación de la inocuidad de las pellas obtenidas en los dos experimentos se resume en la Tabla 5, donde se puede observar que en el recuento de microorganismos no se detectó la presencia de bacterias perjudiciales como
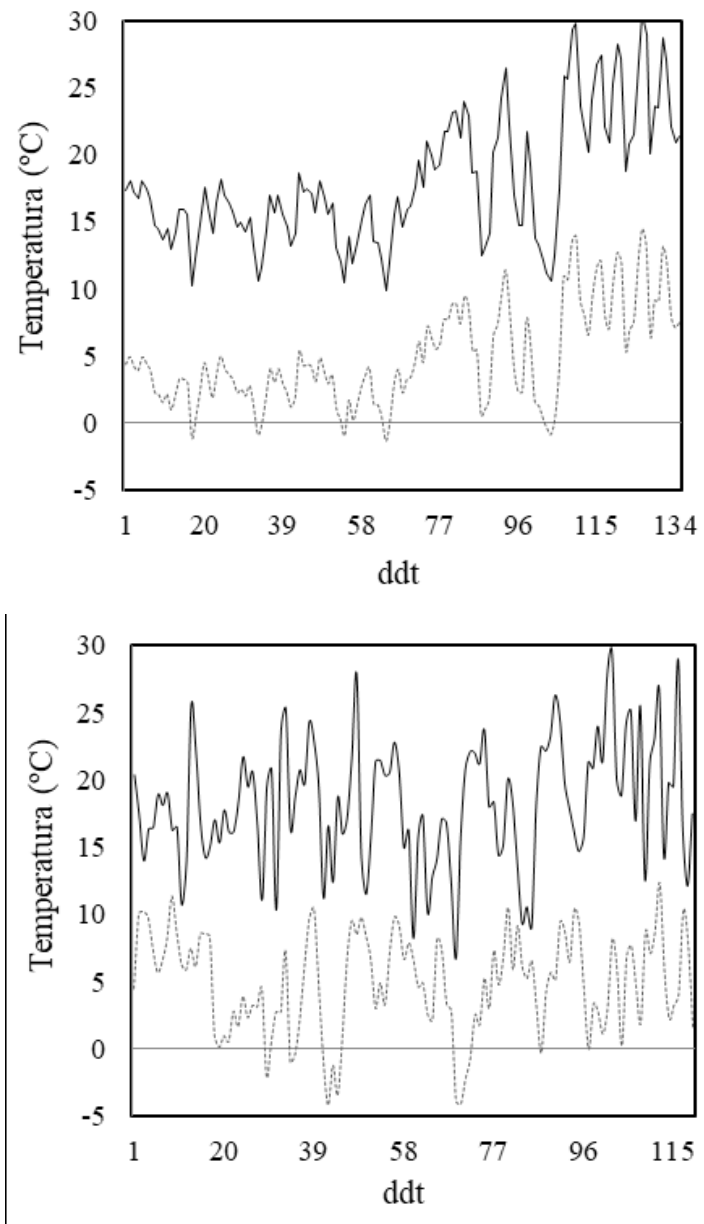

Figura 2. Temperatura mínima y máxima del aire durante el primer (arriba) y segundo (abajo) experimento en los días después del trasplante (ddt).

Escherichia coli y Salmonella sp. sobre las pellas frescas de brócoli. Además, no se detectaron larvas y estructuras parasitarias infectantes, huevos de céstodos, nemátodos y ooquistes que pudieran afectar la salud humana en coincidencia con lo obtenido por Beneduce et al., (2017). Las pellas

Tabla 4. Resultados microbiológicos del EUT y suelo de la parcela a $5 \mathrm{~cm}$ de profundidad.

\section{Muestra}

Cámara concentradora de efluentes crudos

Primera laguna facultativa

Segunda laguna facultativa

EUT para riego

Suelo a $5 \mathrm{~cm}$ de profundidad $\left({ }^{*}\right)$

\section{Unidad}

NMP $100 \mathrm{~mL}^{-1}$ NMP $100 \mathrm{~mL}^{-1}$ NMP $100 \mathrm{~mL}^{-1}$ NMP $100 \mathrm{~mL}^{-1}$ UFC $g^{-1}$
Coliformes totales Coliformes fecales

$2,4 \times 10^{7}$

$7,0 \times 10^{5}$

$1,1 \times 10^{6}$

$4,0 \times 10^{5}$

$4,6 \times 10^{4}$

1.100

$7,5 \times 10^{3}$

240

$9,1 \times 10^{3}$

$1,6 \times 10^{3}$ 
Tabla 5. Resultados de los análisis microbiológicos y parasitológicos de las pellas de brócoli.

\begin{tabular}{lccccc}
\hline \multirow{2}{*}{ Determinación } & \multirow{2}{*}{ Unidad $^{(*)}$} & \multicolumn{2}{c}{ E1 } & E2 & AC \\
\cline { 2 - 5 } & & EUT & AC & EUT & AC \\
\hline Recuento de Coliformes Totales & $\mathrm{UFC} \mathrm{g}^{-1}$ & $1,02 \times 10^{5}$ & $1,6 \times 10^{5}$ & $3,6 \times 10^{5}$ & $4,4 \times 10^{4}$ \\
Recuento de Coliformes Fecales & $\mathrm{Mo} \mathrm{g}^{-1}$ & $<10$ & $<10$ & $<10$ & $<10$ \\
Recuento de Escherichia coli & $\mathrm{Mo} \mathrm{g}^{-1}$ & $<10$ & $<10$ & $<10$ & $<10$ \\
Recuento de Salmonella sp & $\mathrm{A} / \mathrm{P}$ & Ausencia & Ausencia & Ausencia & Ausencia \\
Larvas parasitarias infestantes & $\mathrm{A} / \mathrm{P}$ & Ausencia & Ausencia & Ausencia & Ausencia \\
Huevos de céstodos, nemátodos y & $\mathrm{A} / \mathrm{P}$ & Ausencia & Ausencia & Ausencia & Ausencia \\
ooquistes & & &
\end{tabular}

$\left(^{*}\right)$ UFC $g^{-1}$ : Unidades formadoras de colonia por gramo de muestra; Mo $\mathrm{g}^{-1}$ : Microorganismos por gramo de muestra; A/P: Ausencial Presencia.

fueron aptas para su consumo directo en fresco. Estos hallazgos coinciden con estudios previos que sugieren que el riego con efluentes tratados no implica necesariamente la contaminación de los cultivos (Ramos et al., 2016; Vergine et al., 2017). El sistema de riego por goteo subterráneo contribuyó a la obtención de pellas inocuas, evitando el contacto directo del efluente con el cultivo según lo expresado por Lucas y Alarcón, (2014).

\section{CONCLUSIONES}

Los resultados del presente estudio demostraron que la utilización de efluentes urbanos tratados para riego podría constituir una práctica adecuada en la producción de sistemas hortícolas, reemplazando el uso de aguas de mejor calidad que pueden destinarse para el consumo humano. El aporte de nutrientes al cultivo constribuiría a disminuir el uso de fertilizantes de síntesis química, por lo cual esta práctica podría plantearse como una alternativa complementaria de fertilización. Sin embargo, es sumamente necesario realizar un adecuado tratamiento previo de los efluentes crudos y una correcta aplicación en los cultivos. En este sentido, el uso del riego por goteo subterráneo permitió reducir en gran medida posibles riesgos de contaminanción, logrando un producto de calidad e inocuidad apto para el consumo en fresco.

\section{BIBLIOGRAFÍA}

Ackers, M. L., Mahon, B. E., Leía, E., Goode, B., Damrow, T., Hayes, P. S., Viv, W. F., Rice, D. H., Barrett, T. J., Hutwagner, L., Griffin, P. M. y Slutsker, L. (1998). An outbreak of Escherichia coli 0157:H7 infections associated with leaf lettuce consumption. Journal of Infectious Diseases, 177 (6), 1588-1593. doi: $10.1086 / 515333$

Beneduce, L., Gatta, G., Bevilacqua, A., Libutti, A.,
Tarantino, E., Bellucci, M. y Spano, G. (2017). Impact of the reusing of food manufacturing wastewater for irrigation in a closed system on the microbiological quality of the food crops. International Journal of Food Microbiology, 260, 51-58. doi: 10.1016/j. ijfoodmicro.2017.08.009

Blarasin, M., Cabrera, A. y Matteoda, E. (2014). Aguas subterráneas de la provincia de Córdoba. UniRío. Río Cuarto. Argentina: UniRío Editora.

Cantwell, M. y Suslow, T. (1999). Broccoli. Recommendations for maintaining postharvest quality. University of California. Recuperado de: http:// postharvest.ucdavis.edu/files/259443.pdf

Crespi, R., Camacho, E. y Polo, J. M. (2009). Riego subsuperficial con aguas residuales tratadas. Ingeniería del agua, 16 (2), 145-155. doi: 10.4995/ ia.2009.2946

Crespi, R., Pugliese, M., Grosso, L., Ramos, D., Salusso, F., Soler, E., Solterman, A., Sanchez, A., Rainero, F., Silva, D. y Testa, A. (2012). Generación de biogás y disposición de biosólido. Documento presentado en el XVIII Congreso Argentino de Saneamiento y Medio Ambiente. Buenos Aires, Argentina.

De Chaves, A. B. G. (2016). Estudio del comportamiento de cultivares de brócoli y determinación de las necesidades hídricas y coeficiente de cultivo ' $K c$ '. Tesis de grado. Universidad de La Laguna, Santa Cruz de Tenerife, España. Recuperado de: http:// www.academia.edu/download/48360779/TFG._ Alberto_Beautell.pdf.

Di Benedetto, A. (2005). Manejo de cultivos hortícolas: Bases ecofisiológicas y tecnológicas. Buenos Aires, Argentina: Orientación Gráfica Editora.

Disciglio, G., Gatta, G., Libutti, A., Gagliardi, A., Carlucci, A., Lops, F., Cibelli, F. y Tarantino, A. (2015). Effects of irrigation with treated agro-industrial wastewater on soil chemical characteristics and fungal populations during processing tomato crop cycle. Journal of Soil Science and Plant Nutrition, 15 (3), 765-780. doi: 10.4067/S0718-95162015005000052 
Fasciolo, G., Meca, M. I., Calderon, E. y Rebollo, M. (2005). Contaminación microbiológica en ajos y suelos regados con efluentes domésticos tratados. Rev. Facultad de Ciencias Agrarias U. N. Cuyo, 37 (1), 31-40. Recuperado de: https://www.redalyc.org/ $\mathrm{html} / 3828 / 382838550003 /$

García Alvaro, O. (2012). Criterios modernos para evaluación de la calidad del agua de riego. International Plant Nutrition Institute, (6), 26-34. Recuperado de: http://www.ipni.net/publication/ia-lahp.nsf/0/B3BD6ED 103283DDD85257A2F005EF91B/\$FILE/6\%20Art.pdf

Gil, R. C. y Martelotto, F. E. (1993). El agua edáfica. Guía práctica para su determinación. Córdoba, Argentina: INTA, EEA Manfredi.

González, M. I. y Rubalcaba, S. (2011). Uso seguro y riesgos microbiológicos del agua residual para la agricultura. Revista Cubana de Salud Pública, 37 (1), 61-73. Recuperado de: http:// scielo.sld.cu/scielo.php?script=sci_arttext\&pid =S0864-34662011000100007

Hilborn, E. D., Mermin, J. H., Mshar, P. A., Hadler, J. L., Voetsch, A., Wojtkunski, C., Swartz, M., Mshar, R., Lambert, F. M., Farrar, J. A., Glynn, M. K. y Slutsker, L. (1999). A multistate outbreak of Escherichia coli 0157:H7 infections associated with consumption of mesclun lettuce. Archives of Internal Medicine, 159 (15), 1758-1764. doi:10.1001/archinte.159.15.1758

Isaacs, S., Aramini, J., Ceibin, B., Farrar, J., Ahmed, R., Middleton, D., Howes, H., Chan, E., Chandran, A. U., Harris, L. J., Pichette, S., Campbell, K., Gupta, A., Lior, L. Y., Pearce, M., Clark, C., Rodgers, F., Jameison, F., Brophy, I. y Ellis, A. (2005). An international outbreak of salmonellosis associated with raw almonds contaminated with a rare phage type of Salmonella enteritidis. Journal of Food Protection, 68 (1), 191-198. doi: 10.4315/0362-028X-68.1.191

ISO (2001). Microbiology of food and animal feeding stuffs. Horizontal method for the enumeration of beta-glucuronidase-positive Escherichia coli - Part 2: Colony-count technique at 44 degrees $C$ using 5-bromo- 4 -chloro-3-indolyl beta-D-glucuronide. ISO Norm 16649-2:2001. Ginebra, Suiza: International Standardization Organization.

ISO (2002). Microbiology of food and animal feeding stuffs. Horizontal method for the detection of Salmonella. ISO Norm 6579:2002. Ginebra, Suiza: International Standardization Organization.

Jaramillo, N. J. y Díaz, A. C. (2006). El Cultivo de las Crucíferas. Brócoli, Coliflor, Repollo, Col China. Centro de Investigación La Selva, Rionegro, Antioquia, Colombia: Editorial Litomadrid.

Jay, J. (2002). Microbiología moderna de los alimentos (4a ed.). Zaragoza, España: Editorial Acribia.

Lanchimba Morales, A. K. (2016). Evaluación de dos formulaciones de fertirriego a dos profundidades con fertilización foliar complementaria en la producción de tomate riñón (Lycopersicum esculentum Mill.), var. Micaela bajo invernadero. Tesis de grado. Universidad Central del Ecuador, Pichincha, Ecuador. Recuperado de: http://www.dspace.uce.edu.ec/ handle/25000/7985

Lardizábal, R. y Theodoracopoulos, M. (2008). Manual de Producción de Brócoli. USAID-RED. Proyecto de Diversificacion Económica Rural, Honduras. Recuperado de: http://bvirtual.infoagro.hn/xmlui/ handle/123456789/111

Lucas, F. J. y Alarcón, A. L. (2014). Riego localizado: ventajas e inconvenientes. Recuperado de: http:// www.infoagro.com/riegos/riego_localizado_ventajas_ incovenientes.htm

Moazeni, M., Nikaeen, M., Hadi, M., Moghim, S., Mouhebat, L., Hatamzadeh, M. y Hassanzadeh, A. (2017). Estimation of health risks caused by exposure to enteroviruses from agricultural application of wastewater effluents. Water Research, 125, 104-113. doi: 10.1016/j.watres.2017.08.028

Navarro López, E. R. (2010). Uso de agua residual en la producción de tomate hidropónico en invernadero. Tesis de grado. Universidad Autónoma Chapingo, México. Recuperado de: https://chapingo.mx/ horticultura/pdf/tesis/TESISDCH2010062505123414. pdf

Nieto, S. P., Elizondo, R. M., Angel, R. N., Scoth, M., Fernández, N. H. y Delmar, G. R. (2001). Efecto de las aguas residuales en la calidad y rendimiento en calabacita, Chiconautla, Méx. Documento presentado en XI Congreso Nacional de Irrigación. Simposio 9. Contaminación, Tratamiento y Reuso del Agua. Guanajuato, México.

Orlofsky, E., Bernstein, N., Sacks, M., Vonshak, A., Benami, M., Kundu, A. y Gillor, O. (2016). Comparable levels of microbial contamination in soil and on tomato crops after drip irrigation with treated wastewater or potable water. Agriculture, Ecosystems \& Environment, 215, 140-150. doi: 10.1016/j.agee.2015.08.008

Ramos, D., Salusso, F., Grosso, L., Crespi, R., Pugliese, M., Pautasso, G., Balzola, C. y Alfonso, C. (2016). Producción de ajo bajo riego con efluentes urbanos y aplicación de biosólidos al suelo. Documento presentado en XXXIX Congreso Argentino de Horticultura, Santa Fe, Argentina.

Reinoso, R., Torres, L. A. y Becares, E. (2008). Efficiency of natural systems for removal of bacteria and pathogenic parasites from wastewater. Science of The Total Environment, 395 (2), 80-86. doi: 10.1016/j. scitotenv.2008.02.039

Sáenz Forero, R. (2006). Introducción y uso de aguas residuales tratadas en agricultura y acuicultura. 
Recuperado de: http://www.bvsde.ops-oms.org/ eswww/fulltext/repind53/rys/rys.html

Teuscher, E. (1965). A new single method of examine faeces for diagnosis of helminth disease of rumiants. Zentralblatt für Veterinärmedizin Reihe B, 12 (3), 241248. doi: 10.1111/j.1439-0450.1965.tb01388.x

Tran, Q. K., Schwabe, K. A. y Jassby, D. (2016). Wastewater reuse for agriculture: development of a regional water reuse decision-support model (RWRM) for cost-effective irrigation sources. Environmental Science \& Technology, 50 (17), 9390-9399. doi: 10.1021/acs.est.6b02073

Unión Europea (2017). Nota de la Comisión sobre la Guía para combatir los riesgos microbiológicos en frutas y hortalizas frescas en la producción primaria mediante una buena higiene. 2017/C 163/01. Recuperado de: https://eur-lex.europa.eu/legal-content/ES/TXT/PDF/?u $r i=C E L E X: 52017 X C 0523(03) \& r i d=2$

Vergine, P., Salerno, C., Libutti, A., Beneduce, L., Gatta, G., Berardi, G. y Pollice, A. (2017). Closing the water cycle in the agro-industrial sector by reusing treated wastewater for irrigation. Journal of Cleaner Production, 164, 587-596. doi: 10.1016/j. jclepro.2017.06.239

World Health Organization. (2006). Guidelines for the safe use of wastewater, excreta and greywater - Volume 2. Wastewater use in agriculture. Ginebra, Suiza: World Health Organization. 